\title{
Avaliação da radiodensidade de ionômeros de vidro convencionais e modificados por resina
}

\author{
Radiodensity assessment of conventional and resin-modified \\ glass ionomers
}

\author{
Pedro Henrique Cabral Oliveira \\ Márcia Regina Cabral Oliveira** \\ Paula Roberta Perondi ${ }^{* * *}$ \\ Jamil Awad Shibli $i^{* * * *}$ \\ Alessandra Cassoni $i^{* * *+*}$ \\ José Augusto Rodrigues
}

\section{Resumo}

Objetivo: os ionômeros de vidro (IVs) devem possuir radiodensidade suficiente para se distinguir cáries secundárias e restaurações. Assim, o objetivo do presente estudo foi comparar a radiodensidade de 11 IVs entre convencionais (CO) e modificados por resina (MR), tendo como parâmetro uma escala de alumínio. Materiais e método: 12 corpos de prova de cada grupo $(n=12)$ foram confeccionados em matriz de silicone de adição com $1 \mathrm{~mm}$ de espessura. Os IVMRs foram fotoativados com LED. Uma escala de alumínio e corpos de prova aleatorizados foram posicionados sobre filmes radiográficos periapicais expostos a raios X com $60 \mathrm{kV}, 10 \mathrm{~mA}$ por 0,7 s, com distância foco filme de $10 \mathrm{~cm}$. Após revelação, a radiodensidade foi avaliada qualitativamente por dois examinadores calibrados. Resultados: os dados foram analisados pelo teste de Kruskal Wallis $(p<0,05)$ e teste de Dunn. As medianas (mínimo-máximo) foram lonomaster $^{\circledR}$ (Wilcos-CO): 1(1-1)4; Resiglass ${ }^{\circledR}$ (Biodinâmica-MR): $1(1-2)^{A B}$; Maxxion $R^{\circledR}$ (FGM-CO): $1(1-4)^{A B}$; Vidrion $R^{\circledR}$ (SS White-CO): 1(1-5) ${ }^{A B}$; Vitremer ${ }^{\circledR}$ (3M ES$P E-M R): 2(1-4)^{A B C D}$; lonoseal ${ }^{\circledR}$ (Voco- MR): 3(1-3) ${ }^{A B C D}$; Vitrofi $^{\circledR}$ (DFL-CO): 3,5(1-4)BCD; Ketac Fil ${ }^{\circledR}$ (3M ESPE-CO): 2,5(1-4) $)^{C D}$; Riva light Cure ${ }^{\circledR}$ (SDI-MR): 4(1-4) ${ }^{C D}$; Riva
Self Cure ${ }^{\circledR}$ (SDI-CO): 3(3-4)CD; ChemFil Rock ${ }^{\circledR}$ (Dentsply Caulk-CO): 3,5(2-4)D. Conclusão: os ionômeros de vidro estudados apresentam radiodensidade adequada.

Palavras-chave: Cimentos de ionômeros de vidro. Cimentos dentários. Radiografia. Raios X.

\section{Introdução}

A maior parte do tempo clínico utilizado pelo profissional é empregado no diagnóstico de lesões cariosas e avaliação de restaurações existentes ${ }^{1}$. O critério para substituições de restaurações está, em grande parte, baseado na prevenção ou no tratamento de lesões de cárie secundária, pela presença de espaços no interior ou nas interfaces da restauração, bem como na região de forramento, que são detectados por meio de exame radiográfico ${ }^{1-5}$.

Mestrando em Odontologia, área de concentração em Dentística, Centro de Pós-Graduação e Pesquisa CEPPE, Universidade Guarulhos (UnG), Guarulhos, $\mathrm{SP}$, Brasil.

Cirurgiã-dentista graduada pela UnG, aluna do curso de Especialização em Endodontia, UnG, Guarulhos, SP, Brasil.

Mestra em Odontologia, área de concentração em Dentística, UnG, professora do Departamento de Odontologia Restauradora da UNICASTELO, São Paulo, SP, Brasil.

**** Doutor em Periodontia pela FOAr/UNESP, professor adjunto dos cursos de graduação e de pós-graduação, Centro de Pós-Graduação e Pesquisa CEPPE, UnG, Guarulhos, SP, Brasil.

***** Doutora em Odontologia, área de concentração em Dentística, FO/USP, professora adjunta dos cursos de graduação e de pós-graduação, Centro de PósGraduação e Pesquisa CEPPE, UnG, Guarulhos, SP, Brasil.

Doutor.. em Clínica Odontológica, área de concentração em Dentística, FOP/UNICAMP, professor adjunto dos cursos de graduação e de pós-graduação, Centro de Pós-Graduação e Pesquisa CEPPE, UnG, Guarulhos, SP, Brasil. 
O exame radiográfico é indicado para detectar lesões de cáries oclusais, proximais, primárias e secundárias e apresenta-se como um importante método auxiliar, visto que o exame clínico, mesmo em condições ideais, apresenta limitações, principalmente quando existem pontos de contato estabelecidos na região posterior dificultando a inspeção $0^{1,4,6}$. Todavia, um fator que pode confundir o diagnóstico radiográfico é a imagem radiolúcida de um material de base, forramento ou cimentação, que pode ser sugestivo de lesões secundárias ou desadaptações ${ }^{3,6}$.

De acordo com Espelid et al. ${ }^{6}$, materiais restauradores com radiodensidade menor do que o esmalte dentário e a dentina são difíceis de se distinguir de uma lesão de cárie dentária e podem ser confundidos e interpretados como tal lesão $0^{6}$. Dessa forma, para melhorar o diagnóstico radiográfico, o nível de radiopacidade mínimo para um material de uso restaurador deve ser mais elevado do que o da dentina, ou ligeiramente superior do que o do esmalte dentário ${ }^{7}$. No que diz respeito aos materiais restauradores poliméricos, o Conselho de Materiais Dentários, Instrumentos e equipamentos da ADA (1981), na especificação $\mathrm{n}^{\circ} 27$ de $1977^{7}$, recomenda a radiopacidade como um requisito desejável nos materiais, que possibilite a diferença das imagens radiográficas de um material em uma cavidade e de uma lesão de cárie dentária, permitindo, ainda, avaliar contornos, excessos de material, presença de bolhas e de outros defeitos ${ }^{8,9}$

Motivada por tais fatores, a norma ISO 4049:2000 determina que a radiopacidade de um material de cimentação ou forramento deve ser igual ou maior do que a mesma espessura de alumínio, o que representa uma radiodensidade próxima à da mesma espessura de dentina ${ }^{9}$.

Recentemente, foram introduzidos novos materiais no mercado odontológico, que podem ser chamados de ionômeros modificados por resina, ou de resinas modificadas por poliácido, que, dependendo da reação ácido-base, pode ocorrer como parte do processo de polimerização, o qual se dá com ou sem a presença de luz ${ }^{10}$.

As vantagens dos materiais híbridos em relação aos convencionais estão no controle do tempo de trabalho, na menor sensibilidade à umidade, na maior resistência à fratura e à fadiga, na menor solubilidade e numa melhor estética ${ }^{11,12}$. Outras características, como a microinfiltração marginal e de biocompatibilidade, estão atualmente em análise; no entanto, são poucos os relatos sobre a radiopacidade desses novos materiais ${ }^{4,13}$.

Considerando a dificuldade e a necessidade do diagnóstico de lesões de cáries dentárias recorrentes e nas faces proximais, bem como a adequada visualização dos materiais usados como base, este trabalho teve como finalidade comparar a radiodensidade de 11 ionômeros de vidro convencionais (CO) e modificados por resina (MR), tendo como parâmetro uma escala de alumínio.

\section{Materiais e método}

Foram confeccionados 12 corpos de prova para cada grupo experimental $(\mathrm{n}=12)$. Os grupos experimentais foram compostos pelos materiais ionoméricos em 12 níveis: Ionomaster ${ }^{\circledR}$ (Wilcos, Petrópolis, RJ, Brasil), Resiglass ${ }^{\circledR}$ (Biodinâmica, Ibiporã, PR, Brasil), Maxxion $\mathrm{R}^{\circledR}$ (FGM Joinville, SC, Brasil), Vidrion $\mathrm{R}^{\circledR}$ (SS White, Rio de Janeiro, RJ, Brasil), Vitremer $^{\circledR}$ (3M ESPE, Sumaré, SP, Brasil), Ionoseal ${ }^{\circledR}$ (Voco, Porto Alegre, RS, Brasil), Vitrofil ${ }^{\circledR}$ (DFL, Rio de Janeiro, RJ, Brasil), Ketac Fil ${ }^{\oplus}$ (3M ESPE, Sumaré, SP, Brasil), Riva Light Cure ${ }^{\circledR}$ (SDI, Bayswater, VI, Austrália), Riva Self Cure ${ }^{\circledR}$ (SDI, Bayswater, VI, Austrália) e ChemFil Rock ${ }^{\circledR}$ (Dentsply Caulk, Milford, DE, Estados Unidos da América) e estão apresentados na Tabela 1.

Tabela 1 - Grupos experimentais, material, lote, fabricante e tipo

\begin{tabular}{l|c|c|c}
\multicolumn{1}{c|}{ Material } & Lote & Fabricante & Tipo \\
\hline lonomaster $^{\circledR}$ & ADX0971 & Wilcos & CO \\
Resigless $^{\circledR}$ & $307 / 08$ & Biodinâmica & MR \\
Maxion R $^{\circledR}$ & 070211 & FGM & CO \\
Vidrion $^{\circledR}$ & 20311 & SS White & CO \\
Vitremer $^{\circledR}$ & 20080606 & 3M ESPE & MR \\
lonoseal $^{\circledR}$ & 1032024 & Voco & MR \\
Vitrofil $^{\circledR}$ & 10121715 & DFL & CO \\
Ketac Fil $^{\circledR}$ & 332448 & 3M ESPE & CO \\
Riva Self Cure $^{\circledR}$ & 80905111 & SDI & CO \\
Riva light Cure $^{\circledR}$ & J0908103 & SDI & MR \\
ChemFil Rock $^{\circledR}$ & 1005004003 & Dentsply Caulk & CO \\
\hline
\end{tabular}

Os corpos de prova foram confeccionados em matriz de silicone de adição com $1 \mathrm{~mm}$ de espessura. Para ionômeros MR, foi utilizada fotoativação com luz LED (Radii Cal, SDI; $1600 \mathrm{~mW} / \mathrm{cm}^{2}$ ).

Os espécimes foram colocados aleatoriamente em filmes periapicais dentários (Ektaspeed ${ }^{\circledR}$, Eastman Kodak Co., Rochester, NY, EUA), com uma escala de alumínio como controle, com a radiodensidade variando de 1 a 10 . A exposição radiográfica foi feita com raio $\mathrm{x}$ odontológico (Spectro ${ }^{\circledR} 70 \mathrm{X}$ Eletrônico, Dabi Atlante, Ribeirão Preto, SP, Brasil), fixado em $60 \mathrm{kV}$, com uma corrente de $10 \mathrm{~mA} \mathrm{e}$ um tempo de exposição padrão de $0,7 \mathrm{~s}$. A distância foco-filme foi mantida constante a $10 \mathrm{~cm}$.

Os filmes foram processados manualmente ao mesmo tempo, em um tanque com o mesmo titular. Os invólucros opacos foram removidos a partir dos filmes. Estes foram colocados em um suporte, o qual 
foi imerso na solução reveladora durante 1 minuto e, posteriormente, movimentado para cima e para baixo várias vezes para eliminar as bolhas de ar. Depois disso, as películas radiográficas foram enxaguadas em água durante 20 segundos e imersas na solução de fixação durante 10 minutos, para que os cristais de prata não expostos fossem removidos. Após a conclusão do processo de fixação, o suporte com as películas foi imerso em água corrente durante 5 minutos e, depois, secado.

Concluído o processo de revelação e secagem, as radiografias foram digitalizadas, e a radiodensidade foi avaliada qualitativamente por dois examinadores calibrados (Kappa intra e interexaminador maior que 0,83). Estes avaliaram cegamente as radiografias digitalizadas, utilizando monitores com iluminação e contraste padronizados em um quarto escuro.

Os examinadores atribuíram parâmetros de 1 a 10, a partir da área (degrau) mais radiolucente à mais radiopaca, em relação à escala de alumínio ${ }^{3-5,14}$. Os dados foram analisados pelo teste de Kruskal Wallis $(\mathrm{p}<0,05)$ e teste de Dunn.

\section{Resultados}

Os dados foram analisados pelo teste de Kruskal Wallis $(\mathrm{p}<0,05)$ e teste de Dunn. As medianas (mínimo-máximo) e os resultados do teste de Dunn são apresentados na Tabela 2. A menor radiodensidade foi registrada para os materiais Ionomaster ${ }^{\circledR}$, Resiglass $^{\circledR}$, Maxion $\mathrm{R}^{\circledR}$, e Vidrion $\mathrm{R}^{\circledR}$. Por sua vez, Riva Light Cure ${ }^{\circledR}$, ChemFill Rock ${ }^{\circledR}$ e VitroFil ${ }^{\circledR}$ foram os mais radiopacos, enquanto os demais materiais apresentaram uma radiodensidade intermediária.

Tabela 2 - Resultados do teste de Dunn, mediana, valores máximos e mínimos apresentados nos grupos

\begin{tabular}{l|c|c|c|c}
\hline \multicolumn{1}{c|}{ Material } & Tipo & Mediana & Mínima & Máxima \\
\hline lonomaster $^{\circledR}$ & $\mathrm{CO}$ & $1^{\mathrm{A}}$ & 1 & 1 \\
Resiglass $^{\circledR}$ & $\mathrm{MR}$ & $1^{\mathrm{AB}}$ & 1 & 2 \\
Maxion $^{\circledR}$ & $\mathrm{CO}$ & $1^{\mathrm{AB}}$ & 1 & 4 \\
Vidrion R $^{\circledR}$ & $\mathrm{CO}$ & $1^{\mathrm{AB}}$ & 1 & 5 \\
Vitremer $^{\circledR}$ & $\mathrm{MR}$ & $2^{\mathrm{ABCD}}$ & 1 & 4 \\
lonoseal $^{\circledR}$ & $\mathrm{MR}$ & $3^{\mathrm{ABCD}}$ & 1 & 3 \\
Vitrofil $^{\circledR}$ & $\mathrm{CO}$ & $3,5^{\mathrm{ABCD}}$ & 1 & 4 \\
Ketac Fil $^{\circledR}$ & $\mathrm{CO}$ & $2,5^{\mathrm{CD}}$ & 1 & 4 \\
Riva Self Cure $^{\circledR}$ & $\mathrm{CO}$ & $3^{\mathrm{DC}}$ & 3 & 4 \\
Riva light Cure $^{\circledR}$ & $\mathrm{MR}$ & $4^{\mathrm{DC}}$ & 1 & 4 \\
ChemFil Rock $^{\circledR}$ & $\mathrm{CO}$ & $3,5^{\mathrm{D}}$ & 2 & 4 \\
\hline
\end{tabular}

\section{Discussão}

Além da inspeção clínica, a avaliação dos contornos proximais das restaurações e de seus contatos com os dentes adjacentes pode também ser realizada com exames complementares radiográficos. A radiopacidade relativa dos materiais restauradores em relação à estrutura dental permite o diagnóstico de lesões de cárie secundárias, a detecção de vazios, lacunas, bem como excessos de material restaurador na região proximal e cervical.

Assim, recomenda-se que os materiais restauradores sejam radiopacos e que apresentem radiodensidade maior do que o esmalte dentário e a dentina $a^{4,7,14,15}$. De acordo com a norma ISO 4049:2000, um material de cimentação ou para forramento deve ser igual ou maior do que a mesma espessura de alumínio, o que apresentaria uma radiodensidade próxima à da dentina ${ }^{3,10}$. No presente estudo, todos os materiais cumpriram essa regra, ou seja, apresentaram radiopacidade adequada.

Contudo, a radiodensidade do esmalte dental é semelhante a aproximadamente $2 \mathrm{~mm}$ de alumínio $^{3,4}$, e nem todos os materiais apresentaram esse padrão, o que dificultaria o diagnóstico ${ }^{16}$. É impossível para o clínico prever a radiodensidade de materiais restauradores, então, o uso de materiais ionoméricos com uma radiodensidade ligeiramente maior que as estruturas dentais, como a dentina e o esmalte dental, é aconselhável para avaliação clínica bem-sucedida de restaurações e materiais de forramento e lesões de cárie recorrente ${ }^{17}$.

A possibilidade de diagnóstico da lesão cariosa também é fundamental para a prática odontológica e deve ser baseada nas informações coletadas durante o exame clínico e nos vários procedimentos diagnósticos, principalmente os exames radiográfi$\cos ^{17}$. Em especial, o diagnóstico de lesões cariosas recorrentes ou secundárias não é tarefa fácil, pois diferenciar essas lesões de restaurações proximais, com margens morfologicamente imperfeitas e/ou radiolúcidas, é extremamente complexo, sendo, na maioria das vezes, resultado do julgamento e da interpretação de achados radiográficos ${ }^{17}$.

Assim, os materiais ionoméricos que apresentaram mediana de radiodensidade relativa maior do que o alumínio, ou seja, possivelmente maior do que a dentina e mais próximo ao esmalte, como o Vitremer $^{\circledR}$, Ionoseal ${ }^{\circledR}$, Vitrofil ${ }^{\circledR}$, Ketac Fil ${ }^{\circledR}$, Riva Self Cure $^{\circledR}$, Riva Light Cure ${ }^{\circledR}$ e o ChemFil Rock ${ }^{\circledR}$, facilitariam o diagnóstico clínico.

Embora haja protocolos estabelecidos pela ISO $^{9}$ e ANSI/ADA ${ }^{18}$ para a determinação da radiopacidade de biomateriais baseados na utilização de filmes radiográficos, esses métodos não são sempre usados por pesquisadores. O presente estudo utilizou a escala de alumínio como uma referência que transforma as leituras de transmissão de luz na radiografia 
em uma espessura equivalente de alumínio, com a vantagem de apresentar radiodensidade uniforme, diferente do esmalte e da dentina, que podem variar de acordo com a localização ${ }^{14}$. Esse método foi descrito pela primeira vez por Eliasson e Haasken ${ }^{19}$ (1979) e tem algumas vantagens, tais como baixo custo e a possibilidade da observação de diferenças na radiodensidade dos materiais restauradores, além de possibilitar adequada mensuração de tal característica $^{19}$.

Para simular uma situação clínica, utilizou-se exposições nas películas radiográficas com uma distância foco/filme de $10 \mathrm{~cm}$, procedendo-se, ainda, à avaliação por dois examinadores, com o intuito de identificar a radiopacidade relativa dos materiais. Dessa forma, pôde-se verificar uma considerável diferença entre materiais convencionais e modificados por resina, como sugerido por outros estudos ${ }^{3,15}$.

Assim como verificaram Lachowski et al. ${ }^{3}$ (2013), observou-se que os ionômeros de vidro modificados por resinas, tais como Riva Light Cure ${ }^{\circledR} \mathrm{e}$ Ionoseal ${ }^{\circledR}$, apresentaram a maior radiopacidade em relação aos convencionais Vitrofil ${ }^{\circledR}$, Maxxion $\mathrm{R}^{\circledR}$ e Ionomaster ${ }^{\circledR}$. Em relação aos convencionais, o ChemFil Rock $^{\circledR}$, reforçado por zinco, apresentou os melhores valores de radiodensidade. Os ionômeros de vidro modificados por resina Resiglass ${ }^{\circledR}$ e Vitremer ${ }^{\circledR}$ apresentaram-se menos radiopacos que os convencionais Ketac Fill $^{\circledR}$, VitroFil ${ }^{\circledR}$ e Vidrion ${ }^{\circledR}$, mas a grande diferença foi observada entre os dois materiais convencionais Ionomaster $^{\circledR}$ e ChemFil Rock ${ }^{\circledR}$, com o Resiglass ${ }^{\circledR}$, um material modificado por resina que apresenta radiodensidade semelhante ao Maxion $R^{\circledR}$, que é um ionômero convencional.

Diferenças na composição do material restaurador podem influenciar sua radiopacidade ${ }^{19}$. Os primeiros ionômeros de vidro radiopacos foram 0 Miracle Mix e o Cermet $^{\circledR}$, em razão da modificação de suas composições por prata ${ }^{4}$. Fonseca et al. ${ }^{4}$ observaram que a radiopacidade dos cimentos de ionômero de vidro depende de proporção entre a composição orgânica, que parece ser radiolúcida, e a inorgânica radiopaca. Afirmaram, ainda, que, quanto maior o número atômico das partículas inorgânicas, maior será a radiopacidade conforme o bloqueio da passagem dos raios $\mathrm{X}$.

Dessa forma, a radiodensidade dos materiais convencionais pode ser explicada pelo fato de se tratar de cimentos compostos, basicamente, por elementos de baixo peso molecular, como pó de vidro de cálcio, fluoro-alumino-silicato, e uma solução aquosa contendo copolímero de poliacrílico, ácido itacônico e, também, ácido tartárico, resultando em materiais radiolúcidos ${ }^{14,15}$. A adição de elementos de alto número atômico, tais como estrôncio, bário, lantânio, a fusão da prata ao vidro, ou mesmo a mistura de óxido de zinco, podem torná-los radiopa$\cos ^{20}$. Além disso, o aumento da proporção pó/líquido pode resultar no aumento da radiopacidade ${ }^{4}$.
Hitij e Fidler ${ }^{5}$ demonstraram que cimentos de ionômero de vidro convencionais reforçados apresentam radiodensidade relativa a $2 \mathrm{~mm}$ da escala de alumínio, e os modificados por resina, em torno de $3 \mathrm{~mm}$. Os materiais híbridos de ionômeros de vidro e resina foram desenvolvidos por meio da combinação de componentes resinosos com o cimento de ionômero vidro convencional ${ }^{10-12}$, em uma tentativa de compensar as suas deficiências, tais como a radiolucência, resistência mecânica, e para manter as suas características positivas, tais como liberação de fluoreto e adesão química com as estruturas dentais ${ }^{10-12}$. Em relação à melhora da radiopacidade, o presente ensaio apresentou divergências entre esses materiais, tendo o Resiglass $^{\circledR}$ apresentado baixa radiopacidade, possivelmente devido a uma maior concentração de conteúdo orgânico, caracterizado, ainda, por sua baixa viscosidade. No entanto, a radiodensidade não pode ser excessiva, ou irá obscurecer a lesão de cárie dentária adjacente a uma restauração ${ }^{16}$. Os materiais com um alto grau de radiopacidade são ideais, mas são preferíveis os que têm um grau de radiopacidade moderado ${ }^{16}$.

Assim, os materiais apresentados acima, em sua maioria, com $1 \mathrm{~mm}$ de espessura, estão de acordo com a norma ANSI /Especificação ADA nº 277, embora apresentem uma variação de radiodensidade entre materiais $\mathrm{CO}$ e MR.

\section{Conclusão}

De acordo com os resultados deste estudo, verificou-se que os cimentos de ionômero de vidro estudados apresentam radiodensidade adequada.

\section{Abstract}

Objective: glass ionomers (GIs) must have enough radiodensity to be distinguished from secondary caries and restorations. Thus, this study aimed to compare the radiodensity of 11 conventional (CO) and resin-modified (RM) Gls, with an aluminum scale as parameter. Materials and method: Twelve specimens from each group $(n=12)$ were made with a silicone matrix and additional $1 \mathrm{~mm}$ in thickness. The RMGls were photoactivated with LED. An aluminum scale and the randomized specimens were positioned on periapical radiographic films and exposed to $60 \mathrm{kV} X$-rays, $10 \mathrm{~mA}$ for $0.7 \mathrm{~s}$, and a $10 \mathrm{~cm}$ focus film distance. After film development two calibrated examiners qualitatively assessed radiodensity. Results: data were analyzed by the Kruskal Wallis test $(p<0.05)$, and the Dunn test. The medians (minimum-maximum) were lonomaster ${ }^{T M}$ (Wilcos-CO): 1(11)A; Resiglass ${ }^{T M}$ (Biodynamic-RM): 1(1-2)AB; Maxxion $R^{T M}$ (FGM-CO): 1(1-4)AB; Vidrion $R^{T M}$ (SS White-CO): 1(1-5)AB; Vitremer ${ }^{T M}$ (3M ESPE-RM): 2(1-4)ABCD; lonoseal ${ }^{T M}$ (Voco-RM) 3(1-3)ABCD; VitrofilTM (DFL-CO): 3.5(1-4)BCD; Ketac FilTM (3M ESPE-CO): 2.5(1-4)CD; Riva light Cure ${ }^{T M}$ (SDI-RM): 4(1-4)CD; Riva Self Cure ${ }^{T M}$ 
(SDI-CO): 3(3-4)CD; Chemfil Rock ${ }^{T M}$ (Dentsply Caulk-CO): 3.5(2-4)D. Conclusion: the tested glass ionomers presented adequate radiodensity.

Keywords: Glass lonomer Cements. Dental Cements. Radiography. X-Rays.

\section{Referências}

1. Pekkan G, Ozcan M. Radiopacity of different resin-based and conventional luting cements compared to human and bovine teeth. Dent Mater J 2012; 31(1):68-75.

2. Feitosa FA, Oliveira M, Rodrigues JA, Cassoni A, Reis AF. Comparação da radiodensidade de materiais cimentantes. Rev Odontol UNESP 2011; 40(6):285-9.

3. Lachowski KM, Botta SB, Lascala CA, Matos AB, Sobral MA. Study of the radio-opacity of base and liner dental materials using a digital radiography system. Dentomaxillofac Radiol 2013; 42(2):20120153. doi:10.1259/dentr.20120153.

4. Fonseca RB, Branco CA, Quagliatto PS, Gonçalves Lde S, Soares CJ, Carlo HL, et al. Influence of powder/liquid ratio on the radiodensity and diametral tensile strength of glass ionomer cements. J Appl Oral Sci 2010; 18(6):577-84.

5. Hitij T, Fidler A. Radiopacity of dental restorative materials. Clin Oral Investig 2013;17(4):1167-77.

6. Espelid I, Tveit AB, Erickson RL, Keck SC, Glasspoole EA. Radiopacity of restorations and detection of secondary caries. Dent Mater 1991; 7(2):114-7.

7. Council on Dental Materials, Instruments and Equipment of the American Dental Association. The desirability of using radiopaque plastics in dentistry: a status report. J Am Dent Assoc 1981; 102(3):347-9.

8. International Organization for Standardization. ISO 4049 Dentistry-polymer-based filling, restorative and luting materials. Geneva, Switzerland; 1998.

9. McLean JW, Nicholson JW, Wilson AD. Proposed nomenclature for glass-ionomer dental cements and related materials. Quint Int 1994; 25:587-9.

10. Gladys S, van Meerbeek B, Braem M, Lambrechts P, Vanherle G. Comparative physico-mechanical characterization of new hybrid restorative materials with conventional glassionomer and resin composite restorative materials. J Dent Res 1997; 76:883-94.

11. Sidhu SK, Shah PMM, Chong BS, Pitt Ford TR. Radiopacity of resin-modified glass-ionomer restorative cements. Quint Int 1996; 27:639-43.

12. Marouf N, Sidhu SK. A study on the radiopacity of different shades of resin-modified glass-ionomer restorative materials. Oper Dent 1998; 23:10-4.

13. Pekkan G, Ozcan M. Radiopacity of different resin-based and conventional luting cements compared to human and bovine teeth. Dent Mater J 2012; 31(1):68-75.

14. Reis JM, Jorge EG, Ribeiro JG, Pinelli LA, Abi-Rached F de O, Tanomaru-Filho M. Radiopacity evaluation of contemporary luting cements by digitization of images. doi: ISRN Dent 2012; 2012:704246. http://dx.doi.org/10.5402/2012/704246

15. Tsuge T. Radiopacity of conventional, resin-modified glass ionomer, and resin-based luting materials. J Oral Sci 2009; 51(2):223-30.
16. Espelid I, Tveit AB. A comparison of radiographic occlusal and approximal caries diagnoses made by 240 dentists. Acta Odontol Scand 2001; 59:285-9.

17. ADA Division of Science. ADA Council on Scientific Affairs. Resin-based composites. J Am Dent Assoc 2003; 134:510-2.

18. Eliasson ST, Haasken B. Radiopacity of impression materials. Oral Surg Oral Med Oral Pathol 1979; 47:485-91.

19. Hara AT, Serra MC, Haiter-Neto F, Rodrigues AL Jr. Radiopacity of esthetic restorative materials compared with human tooth structure. Am J Dent 2001; 14(6):383-6.

\section{Endereço para correspondência:}

José Augusto Rodrigues

Programa de Pós-graduação em Odontologia -

Universidade Guarulhos - UnG

Praça Tereza Cristina, 229 - Térreo - Centro

07023-070 Guarulhos, SP

Fone/fax: 55 (11) 2441-3670

E-mail: jrodrigues@prof.ung.br ou guto_jar@ yahoo.com

Recebido: 31/07/2013. Aceito: 18/09/2013. 